\title{
Prospective Assessment of Maximum Protrusion in Patients Wearing a Mandibular Advancement Device
}

\author{
Jean-François Masse, DMD, MSc; Audrey Bellerive, DMD, MSc (Ortho); Frédéric Sériès, MD; Laurie St-Pierre \\ Universite Laval, Quebec City, Quebec, Canada
}

\begin{abstract}
STUdy ОвJестіves: Obstructive sleep apnea (OSA) is a sleep-related disorder with high prevalence in our society. The gold standard for the treatment of sleep apnea is continuous positive airway pressure (CPAP). Given the low adherence rate of patients on CPAP treatment, interest has turned toward dental appliances, commonly called mandibular advancement devices (MAD). The aim of this study was to determine whether patients' maximum protrusion could be increased after wearing a MAD for 3 months, such that patients with weak protrusion (who could at first be withdrawn from treatment) could be eligible for this treatment.

MethoDs: The experimental group included 30 patients in whom OSA was diagnosed by polysomnography. Patients were matched with 30 healthy control patients without OSA. The experimental group wore a MAD for 90 days whereas the control group received no treatment. Both groups underwent two assessments of mandibular borderline movements (protrusion, maximum opening, and left and right lateral movement) at baseline and after 90 days with computerized mandibular scanning.

Results: Results showed that wearing the MAD did not cause greater changes in mandibular movements compared to controls. However, in the MAD group, maximum opening decreased, lateral movement increased, and maximum protrusion increased. Patients with weak maximum protrusion at baseline showed increased maximum protrusion.

Conclusions: Further studies are needed to assess changes in maximum protrusion in patients with weak maximum protrusion. Nevertheless, the findings provide a deeper understanding of the effects of MAD wear on jaw movements, and indicate the potential of the MAD as an effective therapy for patients with maximum protrusion less than $5 \mathrm{~mm}$.

KEYWORDS: dental appliance, mandibular advancement devices, maximum protrusion, obstructive sleep apnea, oral appliance, titration Citation: Masse JF, Bellerive A, Sériès F, St-Pierre L. Prospective assessment of maximum protrusion in patients wearing a mandibular advancement device. Journal of Dental Sleep Medicine. 2018;5(1):11-16.
\end{abstract}

\section{INTRODUCTION}

The American Academy of Sleep Medicine (AASM) defines obstructive sleep apnea (OSA) as a breathing disorder characterized by partial or complete breathing interruptions while asleep associated with lower blood oxygen levels regardless of residual breathing effort. It occurs when the muscles in the back of the throat relax and block the airway. ${ }^{1}$

In 1993, the estimated prevalence of OSA was $2 \%$ for women and $4 \%$ for men, ${ }^{2}$ but current percentages vary widely across populations. Some studies have found rates as high as $23.4 \%$ for women and $49 \%$ for men using polysomnography (PSG). ${ }^{3-5}$ Overweight (body mass index [BMI] $25-29.9 \mathrm{~kg} / \mathrm{m}^{2}$ ), obesity (BMI $\geq 30$ $\left.\mathrm{kg} / \mathrm{m}^{2}\right)$, neck circumference $(>16 \mathrm{in}$, or $40 \mathrm{~cm}$ for women; $>17 \mathrm{in}$, or $43.18 \mathrm{~cm}$ for men), age, family history, sex (higher prevalence in men and menopausal women), ${ }^{6}$ and ethnicity, among other factors, can contribute to the incidence of OSA.,7

The use of continuous airway positive pressure (CPAP), considered the gold standard by the AASM, for treatment of moderate to severe OSA, ${ }^{8}$ increases the residual lung capacity by exerting positive pressure along the respiratory tract from the nose to the alveoli, that is, through the pharyn $\mathrm{x}^{9}$ However, patient nonadherence rates are high $(40 \%$ to $60 \%$, depending on the study), ${ }^{10-12}$ and many complain of secondary effects (eg, unintentional leaks from the mouth and oronasal dryness). ${ }^{13}$ Moreover, despite improved quality of life for patients with high apnea-hypopnea index (AHI),
Batool-Anwar et al. found no improvement for patients with low AHI. ${ }^{14}$

Accordingly, researchers are turning to the mandibular advancement device (MAD) to treat mild to moderate OSA, and for more severe cases when patients refuse CPAP or surgery. ${ }^{15}$ This alternative method can reduce the number of breathing interruptions (by $21 \%$ to $80 \%{ }^{16}$ ) as well as the arousal index ${ }^{17}$ is better tolerated by patients, and is worn for longer periods compared to CPAP devices. ${ }^{11,17}$

Although several studies have examined this topic, weak maximum protrusion excludes a large number of patients from eligibility, ${ }^{18}$ such that they remain untreated. An ongoing study at the Université de Montréal uses a maximum protrusion of less than $5 \mathrm{~mm}$ as an exclusion criterion (personal communication, Dr. Nelly Huynh, PhD, Université de Montréal, 2015) as is the case for the Narval MAD suggested patient selection criteria. ${ }^{19}$ Although Petit et al. questioned MAD effectiveness for patients with less than $6 \mathrm{~mm}$ maximum protrusion, ${ }^{20}$ Marklund et al. obtained significantly higher success rates for patients with greater than $5 \mathrm{~mm}$ maximum protrusion $(P<.01) .{ }^{21}$ Even though we have witnessed a few successful MAD cases in the literature with very little protrusion, the concept itself has never been challenged. Given that the MAD is simple to use, comfortable, and easily portable, ${ }^{22}$ it would be useful to revisit the patient selection criteria and the titration procedure. The objective of this study was therefore to determine whether the maximum protrusion of a patient with OSA could be increased with time using MAD 
titration. To our knowledge, no study to date has addressed this issue. Yet the results could inspire clinicians to review their treatment procedures. The second objective was to assess jaw movements (maximum opening and laterality) in patients fitted with oral appliances in order to predict the effect of long-term MAD wear on the masticatory apparatus. The hypothesis is that by applying mandibular traction nightly, the MAD can induce adaptive changes in the masticatory muscles. This would in turn increase the maximum protrusion by $10 \%$ over a 3 -month titration period in patients with weak maximum protrusion, whereas no change would be observed in the patients in the control group. The null hypothesis is that no significant change will be found between baseline and 3 months of MAD wear.

\section{METHODS}

\section{Patient Recruitment}

This single-center prospective observational clinical study included an experimental group comprising 30 patients presenting with OSA or snoring and who were prescribed MAD treatment by a pulmonologist and seen at the clinic by Dr. Jean-François Masse, in Québec City. An age- and sexmatched control group was included. Data were collected from September 2014 to May 2015.

The following inclusion criteria were used: (1) diagnosis of sleep apnea and/or (2) diagnosis of snoring made by a sleep specialist. Patients were excluded for one or more of the following reasons: (1) absence or refusal of sleep disorder assessment; (2) dental configuration requiring a longer adaptation time; (3) periodontal disease with tooth mobility; (4) opening limit that hinders installation of an adjustable prosthesis; (5) temporomandibular joint disorder with severe pain that could potentially worsen with treatment; (6) medical condition that could require a change in protocol along the way: uncontrolled cardiovascular comorbidity, history of major depression, vehicle accident within the past 12 months, cardiac insufficiency (ejection fraction $<40 \%$ or indication by a general practitioner or cardiologist); (7) history of missed visits; (8) pregnancy during the follow-up period; or (9) jaw displacement after wearing a MAD prior to treatment, resulting in changed baseline position.

The ethics committee of l'Université Laval approved the study protocol (file No.: 2014-055) on May 2, 2014. All 60 patients signed a written informed consent form prior to participating.

\section{Initial Data Collect}

Prior to treatment, a sleep-related disorder had to be diagnosed or patients had to have consulted a pulmonologist (FS) at the Institut universitaire de cardiologie et de pneumologie de Québec (IUCPQ). PSG recordings were analyzed by specialized technicians and the decision to apply therapy was made by a pulmonologist.

At first visit, patients in the control and experimental group responded to a medical questionnaire and underwent a dental examination. Occlusion was measured and jaw muscles were palpated (temporal, masseter, and lateral and medial pterygoid) using a pain-rating scale from 1 to 10 . The protocol was explained to the experimental group, and dental plaster casts were obtained (for the study model and for making the MAD). Protrusion index was then measured according to device type.

Computerized mandibular scanning (CMS) using a K7 system (Myotronics-Noromed, Inc., Kent, Washington, United States) was used. Many clinicians have used this system in private practice, ${ }^{23}$ to calculate maximum protrusion, right and left laterality, and maximum opening to one-tenth of a millimeter. First, patients were asked to reproduce Posselt's Envelope of Motion, starting at maximum intercuspal (MI) position, moving to maximum protrusion, and returning to MI position after reaching maximum opening of the mandible. Patients then moved to maximum right and left laterality and returned to MI position. This procedure was repeated five times and highest measures were recorded. The MAD was then inserted and adjusted, and the patient was instructed on its use. The titration position was set at $50 \%$ to $60 \%$ of the patient's maximum protrusion. Patients were instructed to increase the protrusion in increments of about $1 \mathrm{~mm}$ per week until reaching a comfortable titration endpoint that would be painful if increased further. When the titration period was completed, the effectiveness on upper airway opening was confirmed by PSG at the hospital or by a home sleep apnea test.

\section{Mandibular Advancement Devices}

Three different MAD were used by the experimental group: the EMA (Myerson LLC, Chicago, Illinois, United States), the Narval (ResMed, San Diego, California, United States), and the Somnodent (Somnomed North America, Plano, Texas, United States). The type of MAD used was selected using the following criteria: (1) number of remaining teeth; (2) presence of tooth restorations (crowns, bridges, veneers); and (3) patient's ability (or inability) to breathe through the nose.

\section{EMA}

Twelve patients wore the EMA during titration. The EMA is a removable appliance made of two acrylic bite pads held together on both sides by elastic straps. The straps come in four levels of elasticity and nine lengths. The straps are hooked onto the canines in the upper jaw and to the molars in the lower jaw. They allow personalized adjustment for protrusion and opening during titration: the shorter and firmer the strap, the more the jaw is advanced. The device allows free lateral and protrusion motion. ${ }^{24,25}$

\section{Narval}

Ten patients wore the Narval device, a two-piece orthosis made with a biocompatible polymer material using CAD/ CAM technology. The joint between the two pieces holds the jaw in a forward (protruded) position along the occlusal plane, thereby reducing stress on the temporomandibular joint. It can be adjusted to vary within a $15-\mathrm{mm}$ protrusive range, while allowing free vertical jaw movement. ${ }^{20,26}$

\section{Somnodent (Classic and Flex)}

The Somnodent was given to seven patients. The Somnodent is delivered in two different versions, the Classic and the Flex. The Classic model is held in place using stainless steel ball clasps, whereas the Flex model uses a proprietary soft lining material, the SMH BFlex. 
Table 1-Changes in mandibular movements according to MAD wear and occlusion class.

\begin{tabular}{|c|c|c|c|c|c|c|c|c|}
\hline & \multicolumn{2}{|c|}{ Maximum Opening } & \multicolumn{2}{|c|}{ Maximum Protrusion } & \multicolumn{2}{|c|}{ Right Laterality } & \multicolumn{2}{|c|}{ Left Laterality } \\
\hline & T1 & T2 & T1 & T2 & T1 & T2 & T1 & T2 \\
\hline \multicolumn{9}{|l|}{ Device wear } \\
\hline 7 days $(n=26)$ & $\begin{array}{c}52.1 \\
(37.1 ; 62.4)\end{array}$ & $\begin{array}{c}51.0 \\
(35.3 ; 60.1)\end{array}$ & $\begin{array}{c}10.7 \\
(5,4 ; 16.2)\end{array}$ & $\begin{array}{c}10.8 \\
(5.9 ; 16.8)\end{array}$ & $\begin{array}{c}9.8 \\
(1.4 ; 13.3)\end{array}$ & $\begin{array}{c}10.3 \\
(6.2 ; 13.9)\end{array}$ & $\begin{array}{c}7.4 \\
(1.9 ; 10.6)\end{array}$ & $\begin{array}{c}7.6 \\
(3.8 ; 11.0)\end{array}$ \\
\hline Other $(n=4)$ & $\begin{array}{c}58.3 \\
(55.5 ; 61.3) \\
\end{array}$ & $\begin{array}{c}54.4 \\
(52.0 ; 56.6) \\
\end{array}$ & $\begin{array}{c}10.7 \\
(7.9 ; 11.9)\end{array}$ & $\begin{array}{c}10.8 \\
(8.4 ; 11.7)\end{array}$ & $\begin{array}{c}9.9 \\
(1.4 ; 11.7)\end{array}$ & $\begin{array}{c}11.0 \\
(9.4 ; 12.0) \\
\end{array}$ & $\begin{array}{c}7.1 \\
(5.7 ; 8.1) \\
\end{array}$ & $\begin{array}{c}8.1 \\
(6.8 ; 11.5) \\
\end{array}$ \\
\hline \multicolumn{9}{|l|}{ Occlusion class } \\
\hline Class II div I $(n=8)$ & $\begin{array}{c}50.6 \\
(37.1,60.5)\end{array}$ & $\begin{array}{c}49.1 \\
(35.3 ; 55.6)\end{array}$ & $\begin{array}{c}11.5 \\
(7.2 ; 14.3)\end{array}$ & $\begin{array}{c}11.9 \\
(8.5 ; 14.1)\end{array}$ & $\begin{array}{c}10.5 \\
(7.3 ; 13.3)\end{array}$ & $\begin{array}{c}11.0 \\
(7.9 ; 13.7)\end{array}$ & $\begin{array}{c}7.6 \\
(5.5 ; 9.8)\end{array}$ & $\begin{array}{c}7.7 \\
(5.5 ; 10.7)\end{array}$ \\
\hline Class II div II $(n=8)$ & $\begin{array}{c}50.4 \\
(40.6 ; 61.3) \\
\end{array}$ & $\begin{array}{c}48.7 \\
(39.4 ; 56.6) \\
\end{array}$ & $\begin{array}{c}10.1 \\
(6.7 ; 11.9) \\
\end{array}$ & $\begin{array}{c}9.9 \\
(8.4 ; 11.7) \\
\end{array}$ & $\begin{array}{c}(10.5 \\
(7.6 ; 12.8)\end{array}$ & $\begin{array}{c}10.5 \\
(7.8 ; 12.4)\end{array}$ & $\begin{array}{c}7.7 \\
(5.2 ; 10.6) \\
\end{array}$ & $\begin{array}{c}7.8 \\
(5.9 ; 11.5) \\
\end{array}$ \\
\hline
\end{tabular}

Values presented as average (minimum; maximum). T1 = visit 1. T2 = visit 2 (after 90 days of treatment).

It is bilaterally adjustable to obtain from -0.5 to $5.5 \mathrm{~mm}$ protrusion. The upper (maxillary) wings engage with the lower (mandibular) wings and allow the mouth to open and close freely. ${ }^{27,28}$

\section{Final Data Collection}

Patients in the experimental group only returned to the clinic for a 2-week follow-up and adjustment, and then 1 month later for another follow-up appointment. Data were collected after 3 months using the same CMS system. Initial and final data were stored on the server at Dr. Jean-François Masse's clinic and were transmitted to the statistician at the IUCPQ for blind statistical analysis. The visit protocol was the same for the experimental and control groups. Patients then underwent regular follow-up, according to recommendations by the Canadian Thoracic Society.

\section{Statistical Analysis}

Baseline characteristics are presented as the mean \pm standard deviation. Student $t$ test was performed to compare apnea patients to controls. Mechanical properties of the prostheses were examined using a two-way mixed model. A fixed factor was defined as the comparison between groups according to OSA severity or use of the prosthesis, and the other fixed factor was related to the measurements taken at two different visits with an interaction term between fixed factors. The other fixed factor was analyzed as a repeated-measure factor using an unstructured covariance matrix. The univariate normality assumption was verified with the Shapiro-Wilk tests on the error distribution from the statistical model after a Cholesky factorization. The Brown and Forsythe variation of the Levene test statistic was used to verify the homogeneity of variances. The results were considered significant at $P \leq .05$. All analyses were conducted using SAS v9.4 (SAS Institute, Inc., Cary, North Carolina, United States).

\section{RESULTS}

\section{Sample}

In all, 127 patients were assessed in this study. Of these, 60 were retained, comprising an experimental group $(n=30)$ and a control group $(n=30)$. Of the excluded patients, 67 participated in the study but underwent the final assessment more than 3 months later. The experimental group included 16 men and 14 women, and the control group included 17 men and 13 women. Average age for the experimental group was $48.6 \pm 10.3$ years, and $51.9 \pm 10.0$ years for controls.

Statistical analyses were performed, taking into account the influence of characteristics liable to affect the jaw movements of patients in the experimental group: (1) nightly MAD wear (or not); (2) size of overbite; and (3) occlusion class (Table 1). Statistical tests were performed to determine whether these parameters had a statistically significant effect on maximum protrusion, maximum opening, and laterality.

\section{MAD Wear}

Patients were divided into two wear groups: (1) those who wore the MAD every night (7 nights/wk); and (2) those who did not meet this criterion (other: 4 to 5 nights/wk, alternated with CPAP or nonwear for a certain period). We noted a reduction in maximum opening for most patients who wore the MAD $(n=20)$. However, this reduction appeared to be similar for both wear groups, and MAD wear did not have a statistically significant effect on the results $(P>.05)$. We also observed a mean increase in protrusion of $0.95 \pm 0.69 \mathrm{~mm}$ in patients who showed a positive response $(n=17)$, varying from 0.1 to $2.1 \mathrm{~mm}$. The number of nights of MAD wear per week did not appear to affect the results $(P>.05)$. For right and left laterality, we observed an increase $(0.5 \pm 0.2 \mathrm{~mm})$ for treatment-adherent patients, and a $(1.6 \pm 1.0 \mathrm{~mm})$ increase for less adherent patients. Most patients in the experimental group $(\mathrm{n}=26)$ showed at least increased protrusion movement $(P<.0001)$.

\section{Overbite}

The statistical results indicated that the variation in jaw movement amplitude was not related to overbite $(P>.05)$.

\section{Occlusion Class}

Occlusion class was determined for the experimental group using model analysis. We observed a reduction in maximum opening between the two visits: $1.4 \mathrm{~mm}$ for Class $\mathrm{I}, 1.5 \mathrm{~mm}$ 


\section{Figure 1}

Change in maximum protrusion after wearing a MAD (experimental group patients)

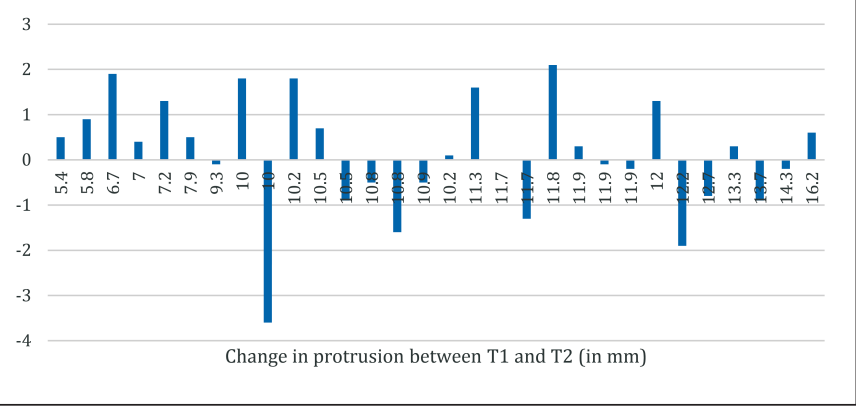

\section{Figure 3}

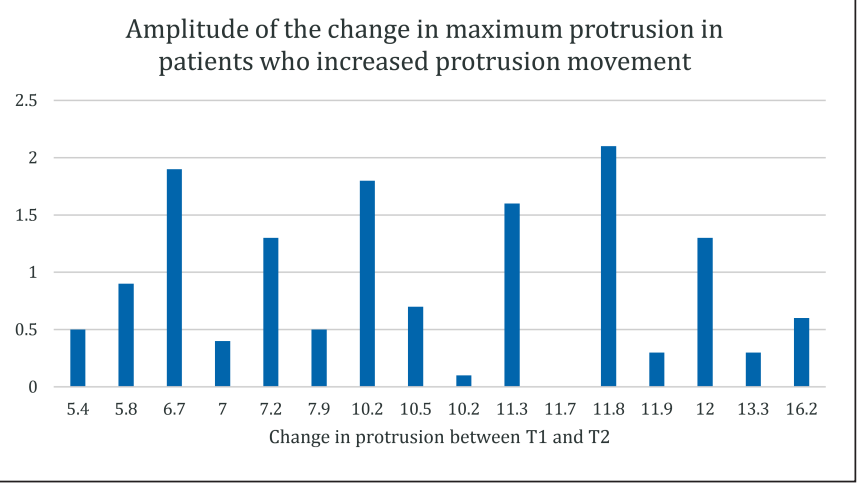

for Class II - division I, and $1.7 \mathrm{~mm}$ for Class II - division II. In addition, protrusion increased for patients with Class I (0.1 $\mathrm{mm})$ and Class II - division I (0.4 mm). Patients with Class II - division II showed reduced maximum protrusion in $75 \%$ of cases, with increased protrusion for $25 \%$. Positive changes in right and left laterality were observed for Class I ( $0.6 \pm 0.5$ $\mathrm{mm})$ and Class II - division I $(0.5 \pm 0.1 \mathrm{~mm})$, but almost no changes for Class II - division II $(0.0 \pm 0.1 \mathrm{~mm})$. However, occlusion class did not have statistically significant effects on these changes $(P>.05)$.

Results showed very little difference in protrusion between experimental and control groups. In fact, due to the large variability in the experimental group, the average protrusion increase was $0.11 \pm 1.27 \mathrm{~mm}$, with $0.66 \pm 1.40 \mathrm{~mm}$ for controls $(P=.1)$.

\section{DISCUSSION}

Patients had good treatment adherence. Most of these patients wore the prosthesis every night, although two wore it only 4 nights/wk and one alternated with CPAP every second night. One patient stopped using the MAD for 42 days and received a final assessment after 90 days of treatment.

To our knowledge, no study to date has addressed jaw movements in patients who wear a MAD. Many studies have established an exclusion criterion of a maximum protrusion of at least $5 \mathrm{~mm}$, but there is no empirical evidence to support this. Considering various protrusions would allow determining changes in protrusion according to patients' initial protrusion (see Figure 1 and Figure 2). In fact, initial

\section{Figure 2}

Change in maximum protrusion for the 6 patients with the weakest protrusion (5.4 to $7.9 \mathrm{~mm}$ ) and the 6 patients with the greatest protrusion (12.2 to $16.2 \mathrm{~mm}$ )

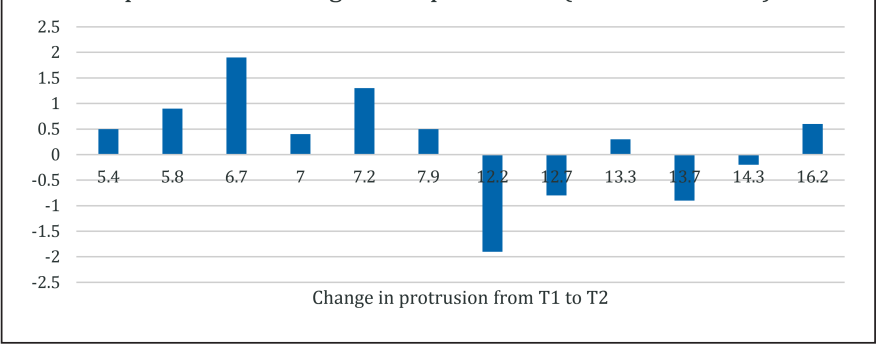

protrusion increased in all patients with weak protrusion at baseline (from 5.4 to $7.9 \mathrm{~mm}$ ), whereas the increase varied in patients with initial protrusion greater than $10.5 \mathrm{~mm}$. For the 17 patients who showed increased protrusion amplitude, the increase varied from 0.1 to $2.1 \mathrm{~mm}$, for an average of 0.95 $\mathrm{mm}$ (Figure 3), whereas 9 others had initial protrusion less than $10 \mathrm{~mm}$. We paid particular attention to this finding, because most of the sample showed positive changes. The ultimate goal was to make patients with maximum protrusion less than $5 \mathrm{~mm}$ eligible for MAD treatment. In fact, the results indicate that maximum protrusion can be increased in patients with weak initial protrusion. However, the inverse dynamic was observed for patients with larger protrusion at baseline: all patients whose protrusion was reduced had a protrusion greater than $9.3 \mathrm{~mm}$ at baseline. Figure 2 shows a comparison between the six patients with the weakest protrusion and the 6 with the greatest protrusion. In addition, the reduction in maximum protrusion was more predominant among patients with Class II - division II occlusion (six reductions out of eight patients in this class). Retroclination of the central upper incisors can hinder protrusion movement and hence reduce the potential to increase. Furthermore, the graphics show that the changes ${ }^{29}$ in protrusion, albeit random, are similar in quantity to those for weaker and greater protrusion. However, the clinical significance differs for the two classes: the $0.5 \mathrm{~mm}$ change for the patient with $5.4 \mathrm{~mm}$ at baseline corresponds to a $9.26 \%$ increase, whereas the $0.6 \mathrm{~mm}$ change for the patient with $16.2 \mathrm{~mm}$ at baseline corresponds to a $3.70 \%$ increase (Figure 3).

Some controls also increased their maximum protrusion during the treatment period. First, we thought that this group would show no change between initial assessment and treatment end, having not worn the MAD. Nevertheless, we observed some increases as well as decreases. The first hypothesis is that this variability could be attributed to factors that were not controlled for, and which should be considered in future studies. For instance, some patients in the control group could have become aware of the expected results at the first visit, and they could have practiced the movements before the second assessment. Could practicing jaw movements without wearing a prosthesis lead to results similar to those for prosthesis wearers? This question merits a separate study. A second hypothesis is that the study may have lacked power: with a $5 \%$ threshold and $80 \%$ power, groups of 92 patients each would 
be required to detect significant between-group differences. In this perspective, our trial would be insufficiently robust to determine whether MAD wear is really effective in increasing jaw motion.

For its part, the reduced maximum protrusion in patients who wore the MAD could have been influenced by several factors. (1) Patient stress has been repeatedly proposed as a contributing factor for increased muscle activity, for example, in the trapezius muscle when using an unfamiliar computer. ${ }^{30}$ Thus, increased muscular activity in the masticatory apparatus (temporal, masseter, and lateral and medial pterygoid) during data collection with the K7-CMS could have contributed to differing results between visits. (2) Muscular pain: wearing a prosthesis that relaxes the muscles for a period of 6 months leads to a substantial change in jaw movement amplitude. ${ }^{31}$ Thus, wearing the MAD could have increased the sensitivity of the masticatory muscles (particularly the masseter and temporal) and reduced their pain tolerance threshold. ${ }^{29}$ (3) Temporomandibular disorders: wearing the MAD stretches the muscle fibers, causing tension in the jaw muscles and the temporomandibular joint. According to the study by Merrill, the tension due to pain in the temporomandibular joint reported by $10 \%$ to $13 \%$ of MAD wearers limits its utility. ${ }^{32}$ However, Gawriolek et al. argue that temporomandibular joint dysfunction is more prevalent among Class I and II patients, ${ }^{31}$ as seen in these patients in the current study, whose maximum protrusion decreased. In these patients, when the advancement was decreased by only $1 \mathrm{~mm}$ during the MAD wear period, the resultant pain was significantly reduced..$^{32}$ (4) Presence of parafunctions: activity such as bruxism, which causes jaw pain, varies over time and could potentially limit jaw movements. ${ }^{33}$

This examination of additional jaw movements sheds light on the fact that MAD wear decreases the maximum opening for patients with all occlusion classes and obtains similar results for patients with good and poorer adherence (see Table 1). These results are not clinically significant, however, and similar results were observed for some patients in the control group. In terms of laterality, most patients showed increases, providing further insight into how jaw movements change with MAD wear, with mainly increased muscular amplitude.

A method to assess patients' potential for positive results using a MAD was commercialized by Zephyr Sleep Technologies: MATRx is a remote-controlled oral appliance titration method that assesses, during a sleep laboratory night, the potential effectiveness of oral appliance therapy and identifies the target protrusive position for the patient. ${ }^{34}$ Our results call into question the criterion of patients being considered unresponsive if they have weak protrusion at baseline and incompletely open airways during the night. These patients have the potential to increase their mandibular advancement over the first months of MAD wear. Our findings also call into question the accuracy of results on patients with limited maximum protrusion.

In future studies, it would be useful to recruit patients with weaker initial protrusion, at less than $7 \mathrm{~mm}$. The low number of these participants in our study makes our results, while promising, irreproducible. It would also be useful to review the patient titration protocol. For example, it could be determined whether an approximate $50 \%$ advancement would be sufficient to obtain satisfactory results, ${ }^{15}$ would help prevent muscular pain for some patients, and would benefit only patients with particular occlusion types. Patients with Class III occlusion could also be included. Given the low prevalence of Class III occlusion in the overall population and the low prevalence in the apnea population in particular, our sample did not contain any such patients. These patients could have worn the MAD for longer than 3 months to verify whether muscular adaptation improved with longer MAD wear. Finally, we should include about 100 patients per group to obtain acceptable statistical power.

To summarize, the patients who participated in this study received treatment that they continued after the final data collect, without further visits or changes to the titration protocol. This study provides a deeper understanding of patients' capacities after MAD wear, and highlights the potential of this treatment for patients with maximum protrusion less than $5 \mathrm{~mm}$.

\section{CONCLUSIONS}

In summary, this study suggests (1) the changes in maximum protrusion, maximum opening, and laterality cannot be attributed to factors such as device wear, overbite, and occlusion class; (2) device wear induces changes in the range of jaw movements; and (3) patients with weaker maximum protrusion at baseline are liable to increase it by wearing a MAD, such that patients with less than the minimum required protrusion could be eligible for treatment with the MAD.

\section{ABBREVIATIONS}

\section{AASM, American Academy of Sleep Medicine}

AHI, apnea-hypopnea index

BMI, body mass index

CAD/CAM, computer-aided design/computer-aided manufacturing

CPAP, continuous positive airway pressure

MAD, mandibular advancement device

MI, maximum intercuspal

OSA, obstructive sleep apnea

PSG, polysomnography

\section{REFERENCES}

1. American Academy of Sleep Medicine. Obstructive Sleep Apnea [factsheet]. https://aasm.org/resources/factsheets/sleepapnea.pdf. Accessed December 18, 2017.

2. Young T, Palta M, Dempsey J, Skatrud J, Weber S, Badr S. The occurrence of sleep-disordered breathing among middle-aged adults. N Engl J Med .1993;328(17):1230-1235.

3. Heinzer R, Vat S, Marques-Vidal P, et al. Prevalence of sleepdisordered breathing in the general population: the HypnoLaus study. Lancet Respir Med. 2015;3(4):310-318.

4. Verhulst SL, Van Gaal L, De Backer W, Desager K. The prevalence, anatomical correlates and treatment of sleep-disordered breathing in obese children and adolescents. Sleep Med Rev. 2008;12(5):339-346.

5. Marcus CL. Sleep-disordered breathing in children. Am J Respir Crit Care Med. 2001;164(1):16-30. 
6. Kapsimalis F, Kryger M. Sleep breathing disorders in the U.S. female population. J Womens Health (Larchmt). 2009;18(8):1211-1219.

7. Cao MT, Guilleminault C, Kushida CA. Clinical Features and Evaluation of Obstructive Sleep Apnea and Upper Airway Resistance Syndrome. In: Kryger MH, Roth T, Dement WC, eds. Principles and Practice of Sleep Medicine. 5th ed. St. Louis, MO: Elsevier Saunders; 2011:1206-1218.

8. Conley RS. Management of sleep apnea: a critical look at intra-oral appliances. Orthod Craniofac Res. 2015;18(Suppl 1):83-90.

9. Phillips BA, Kryger MH. Management of Obstructive Sleep ApneaHypopnea Syndrome. In: Kryger MH, Roth T, Dement WC, eds. Principles and Practice of Sleep Medicine. 5th ed. St. Louis, MO: Elsevier Saunders; 2011:1278-1293.

10. Kribbs NB, Pack AI, Kline LR, et al. Objective measurement of patterns of nasal CPAP use by patients with obstructive sleep apnea. Am Rev Respir Dis. 1993;147(4):887-895.

11. Phillips CL, Grunstein RR, Darendeliler MA, et al. Health outcomes of continuous positive airway pressure versus oral appliance treatment for obstructive sleep apnea: a randomized controlled trial. Am J Respir Crit Care Med, 2013;187(8):879-887.

12. Engleman HM, Wild MR. Improving CPAP use by patients with the sleep apnoea/hypopnoea syndrome (SAHS). Sleep Med Rev. 2003;7(1):81-99.

13. Janson C, Noges E, Svedberg-Randt S, Lindberg E. What characterizes patients who are unable to tolerate continuous positive airway pressure (CPAP) treatment? Respir Med. 2000;94(2):145-149.

14. Batool-Anwar S, Goodwin JL, Kushida CA, et al. Impact of continuous positive airway pressure (CPAP) on quality of life in patients with obstructive sleep apnea (OSA). J Sleep Res. 2016;25(6):731-738.

15. Bartolucci ML, Bortolotti F, Raffaelli E, D’Anto V, Michelotti A, Alessandri Bonetti G. The effectiveness of different mandibular advancement amounts in OSA patients: a systematic review and metaregression analysis. Sleep Breath. 2016;20(3):911-919.

16. Serra-Torres S, Bellot-Arcis C, Montiel-Company JM, Marco-Algarra J, Almerich-Silla JM. Effectiveness of mandibular advancement appliances in treating obstructive sleep apnea syndrome: a systematic review. Laryngoscope. 2016;126(2):507-514.

17. Okuno K, Sato K, Arisaka T, et al. The effect of oral appliances that advanced the mandible forward and limited mouth opening in patients with obstructive sleep apnea: a systematic review and meta-analysis of randomised controlled trials. J Oral Rehabil. 2014;41(7):542-554.

18. Shen HL, Wen YW, Chen NH, Liao YF. Craniofacial morphologic predictors of oral appliance outcomes in patients with obstructive sleep apnea. J Am Dent Assoc. 2012;143(11):1209-1217.

19. Narval CC: The first CAD/CAM custom made MRD. Great Lakes Orthodontics website. https://www.greatlakesortho.com/resourcecenter/dental-sleep-center/narval-cc/. Published 2012. Accessed December 6, 2017.

20. Petit FX, Pepin JL, Bettega G, Sadek H, Raphael B, Lévy P. Mandibular advancement devices: rate of contraindications in 100 consecutive obstructive sleep apnea patients. Am J Respir Crit Care Med. 2002;166(3):274-278.

21. Marklund M, Franklin KA, Sahlin C, Lundgren R. The effect of a mandibular advancement device on apneas and sleep in patients with obstructive sleep apnea. Chest. 1998;113(3):707-713.

22. Ngiam J, Balasubramaniam R, Darendeliler MA, Cheng AT, Waters $\mathrm{K}$, Sullivan CE. Clinical guidelines for oral appliance therapy in the treatment of snoring and obstructive sleep apnoea. Aust Dent $J$. 2013;58(4):408-419.

23. K7 Evaluation System: The Comprehensive Instrument for Precise Occlusal Evaluation. Myotronics website. http://www.myotronics. com/products/k7-evaluation-system/. Accessed December 6, 2017.
24. Henke KG, Frantz DE, Kuna ST. An oral elastic mandibular advancement device for obstructive sleep apnea. Am J Respir Crit Care Med. 2000;161(2 Pt 1):420-425.

25. EMA Sleep Appliance. Protec Dental Laboratories LTD website. http://www.protecdental.com/product/ema-sleep-appliance. Published 2016. Accessed December 6, 2017.

26. Narval CC: Discover the Narval difference. ResMed website. https:// www.resmed.com/us/en/healthcare-professional/products/dental/ narval.html. Published 2010. Accessed December 6, 2017.

27. SomnoDent Oral Device Product Information. SomnoMed website. https://somnomed.com/en/dentists/somnodent-product-information. Accessed December 18, 2017.

28. Oral Appliances Side-by-Side Comparison Guide (December 2016). Sleep Review website. http://www.sleepreviewmag.com/2017/01/ oral-appliances-guide-dec-2016. Published January 3, 2017. Accessed December 18, 2017.

29. Alessandri-Bonetti G, Bortolotti F, Bartolucci ML, Marini I, D'Anto V, Michelotti A. The effects of mandibular advancement device on pressure pain threshold of masticatory muscles: a prospective controlled cohort study. J Oral Facial Pain Headache. 2016;30(3):234-240.

30. Taib MF, Bahn S, Yun MH. The effect of psychosocial stress on muscle activity during computer work: comparative study between desktop computer and mobile computing products. Work. 2016;54(3):543-555.

31. Gawriolek K, Azer SS, Gawriolek M, Piotrowski PR. Mandibular function after Myorelaxation Therapy in temporomandibular disorders. Adv Med Sci. 2015;60(1):6-12.

32. Merrill RL. Temporomandibular disorder pain and dental treatment of obstructive sleep apnea. Dent Clin North Am. 2012;56(2):415-431.

33. Fernandes G, Franco-Micheloni AL, Siqueira JT, Goncalves DA, Camparis CM. Parafunctional habits are associated cumulatively to painful temporomandibular disorders in adolescents. Braz Oral Res. 2016;30.

34. Product Series. Zephyr Sleep Technologies website. https://www.zephyrsleep.com/products/product-series. Accessed December 18, 2017.

\section{ACKNOWLEDGMENTS}

The authors thank the fonds Émile Beaulieu for funding this study and the participants for their contribution and support. They also thank Serge Simard, statistician at the IUCPQ, for his contribution, and Margaret McKyes for translating the manuscript into English from the original French.

\section{SUBMISSION \& CORRESPONDENCE INFORMATION}

Submitted for publication July 15, 2017

Submitted in final revised form September 8, 2017

Accepted for publication October 3, 2017

Address correspondence to: Audrey Bellerive, Pavillon de médecine dentaire, local 5560; Tel: 418 656-2131, poste 5134; Email: audrey. bellerive@fmd.ulaval.ca

\section{DISCLOSURE STATEMENT}

Work for this study was performed at Centre Dentaire Jean-François Masse (Québec), Faculté de Médecine dentaire de l'Université Laval (Québec), and Institut universitaire de cardiologie et pneumologie de Québec (IUCPQ). This study was funded by the fonds Émile-Beaulieu of the faculté de Médecine Dentaire de l'Université Laval. The authors report no conflicts of interest. 